\section{The prevalence of advanced loss of periodontal attachment in two New Mexico populations}

\author{
Ismail AI, Eklund SA, Striffler DF and Szpunar SM: The prevalence of advanced \\ loss of periodontal attachment in two New Mecixo populations. Journal of Periodontal \\ Research 1987; 22: 119-124.
}

This paper describes the prevalence of destructive periodontal disease, in two samples of New Mexico adults, examined in two separate studies 26 years apart, by analyzing the occurrence of advanced loss of periodontal attachment (7.0 or more $\mathrm{mm}$ ) in relation to selected dental and social factors. The first group comprised 1976 dentate adults aged 17-46 yr who were examined in 1958-59. The second study, conducted in 1984, included 372 dentate adults aged 27-74 yr. In the 1958 study, the prevalence of advanced loss of attachment was significantly associated with age, gender, race, education, plaque scores, and calculus scores. In 1984, the prevalence of advanced loss was significantly associated with age, education, and the presence of plaque. The analysis of loss of periodontal attachment data from both studies showed that 0.3 and $1.5 \%$ of all teeth present in the mouth in persons examined in 1958 between the ages of 17-26 and 27-46 $\mathrm{yr}$, respectively, had advanced loss of attachment. In the 1984 sample, 1.5 and $6.7 \%$ of the 12 tooth sites examined in persons $27-46$ and 47-74 yr had advanced loss of periodontal attachment, respectively. These observations are in agreement with recent epidemiological studies of the prevalence and distribution of advanced loss of periodontal attachment.
Amid I. Ismail', Stephen A. Eklund², David F. Striffler ${ }^{2}$ and Susan $M$. Szpunar ${ }^{2}$

'McGill University, Montreal, Quebec,

${ }^{2}$ The University of Michigan, School of Public Health, Ann Arbor, Mi, U.S.A.

\section{Introduction}

Most epidemiological studies of periodontal disease in the United States have been cross-sectional in design and have used indices that were not designed to provide information on the intraoral severity and distribution of the disease (National Center for Health Statistics 1965, 1979). In the Health Examination Survey (HES), and the first National Health and Nutrition Examination Survey (NHANES I), both conducted by the National Center for Health Statistics (NCHS) in 1960-62, and 1971-74, respectively, the prevalence of periodontal disease in American adults was measured using the Periodontal Index (Russell 1956). Analyses of data abstracted from the 1960-62 survey found that $46 \%$ of those adults aged $65-74$ had periodontal pockets (a pocket was diagnosed when the distance from the free gingival margin to the base of the gingival crevice was greater than 3.0 $\mathrm{mm}$ ). In 1971-74, about $50 \%$ of those adults aged 65-74 were affected by pocketing (National Center for Health
Statistics 1979). Further analyses of the prevalence of loss of periodontal attachment (measured from the cementoenamel junction to the bottom of the pocket) were not carried out because the PI does not collect these types of data.

A secondary analysis of the NHANES I data, however, showed that half of the dentate Americans aged 65-74 were free from periodontal pocketing as measured according to the PI criteria (Burt, Ismail \& Eklund 1985). This finding confirms those of recent studies in the United States and other countries (Hughes, Rozier \& Ramsey 1982, Beck et al. 1984, Hugoson \& Jordan 1982, Reddy et al. 1985). Also, recent studies found that destructive periodontal disease (presence of pockets equal to or deeper than $6.0 \mathrm{~mm}$ ) in adults is less frequent than previously thought (Beck et al. 1984, Reddy et al. 1985, Hugoson \& Jordan 1982, Ismail et al. 1986, Baelum et al. 1986). In Iowa, for example, only $2.1 \%$ of adults $65 \mathrm{yr}$ or older had pockets of $6 \mathrm{~mm}$ or deeper (Beck et al. 1984). These studies, as well as the current questioning of the PI and other periodontal indices (Burt et al. 1985) and the lack of knowledge about the natural history of the disease, suggest that a fresh approach to the collection and analysis of periodontal disease data is required.

The purpose of this paper is to describe the prevalence of destructive periodontal disease in two samples of New Mexico adults, examined in two separate studies 26 yr apart, by analyzing the occurrence of advanced loss of periodontal attachment ( 7.0 or more $\mathrm{mm}$ ), in relation to selected dental and social factors. The first group included 1976

Table 1. Number of participants in the 1958 New Mexico survey of adults and the 1984 Deming-Lordsburg survey of adults, by age and gender

\begin{tabular}{lcccc}
\hline & \multicolumn{4}{c}{ Age group in years } \\
& \multicolumn{2}{c}{1958} & \multicolumn{2}{c}{1984} \\
Gender & $17-26$ & $27-46$ & $27-46$ & $47-74$ \\
\hline Male & 335 & 643 & 83 & 47 \\
Female & 372 & 626 & 169 & 82 \\
Total & 707 & 1269 & 252 & 129 \\
\hline
\end{tabular}


dentate adults, aged 17 to $46 \mathrm{yr}$, examined from May, 1958 to December, 1959 (Sheiham \& Striffler 1970a, b). The second study was conducted in 1984 and included 372 dentate adults aged 27 to $74 \mathrm{yr}$ who were long-term residents of Lordsburg and Deming, New Mexico.

\section{Material and Methods \\ The 1958 New Mexico survey of adults}

In 1958, a state-wide survey of oral health status was conducted by the New Mexico Department of Public Health (now known as the New Mexico Department of Health and Environment). The main purpose of the survey was to provide data on the prevalence of different oral conditions in New Mexico adults between 17 and $46 \mathrm{yr}$ of age. The oral conditions measured included dental caries, periodontal disease using both the PI (Russell 1956) and the PDI (Periodontal Disease Index)(Ramfjord 1959), oral hygiene status using the Oral Hygiene Index later described by Greene \& Vermillion (1960), fluorosis (Dean 1942), tooth attrition, mobility of teeth, drifting of teeth, open contacts, and other conditions.

Table 2. Mean number of teeth per person with specified amount of loss of periodontal attachment, by age, 1958 New Mexico survey of adults

\begin{tabular}{cccccc}
\hline Age & $\mathrm{N}$ & $1-3 \mathrm{~mm}$ & $4-6 \mathrm{~mm}$ & $7+\mathrm{mm}$ & $\begin{array}{c}\text { Mean no. of } \\
\text { teeth examined } \dagger\end{array}$ \\
\hline $17-26$ & 707 & 7.68 & 0.27 & 0.07 & 26.42 \\
$27-46$ & 1269 & $14.16^{*}$ & $2.12^{*}$ & $0.38^{*}$ & 24.79
\end{tabular}

* Mean loss of those aged 27-46 is significantly higher than those aged 17-26 yr, p $<0.0001$. †Includes teeth with no loss of periodontal attachment.

Table 3. Percentage of persons with at least one site with a specified amount of loss of periodontal attachment, by selected characteristics, 1958 New Mexico survey of adults

\begin{tabular}{lrrrr}
\hline Characteristic & $\mathrm{N}$ & $1-3 \mathrm{~mm}$ & $4-6 \mathrm{~mm}$ & $7+\mathrm{mm}$ \\
\hline Age & & & & \\
$17-26$ & 707 & 87.4 & 11.7 & 2.4 \\
$27-46$ & 1269 & 99.1 & 49.1 & 14.1 \\
Gender & & & & \\
Male & 978 & 95.3 & 42.4 & 13.2 \\
Female & 998 & 94.5 & 29.2 & 6.7 \\
Ethnic Group & & & & \\
Spanish & 679 & 97.2 & 42.9 & 13.7 \\
Indian & 76 & 100.0 & 43.4 & 11.8 \\
Black & 37 & 94.6 & 43.2 & 18.9 \\
Anglo & 1177 & 93.3 & 31.0 & 7.4 \\
Education (yr) & & & & \\
0-8 & 366 & 98.1 & 52.5 & 17.8 \\
9-11 & 417 & 93.3 & 38.6 & 10.6 \\
12 & 714 & 96.4 & 31.8 & 8.4 \\
College & 471 & 91.5 & 26.1 & 5.7 \\
\hline
\end{tabular}

the use of the PI, PDI, and OHI by the originators of the indices. Loss of periodontal attachment was measured as described by Ramfjord - that is, from the cementoenamel junction to the base of the pocket using a Michigan \#0 probe. Measurement of loss of attachment was carried out for all teeth present in the mouth, excluding third molars. Four measurements were obtained from the distal, buccal, mesial, and lingual tooth surfaces. The largest measurement was selected to represent the tooth.

Measurement of oral hygiene status was carried out using the Oral Hygiene Index (OHI) scoring method developed by Greene ("Greene, J. C. A system for classifying oral hygiene. Washington, Public Health Service, n.d., 10 p. processed"). Each dental arch was divided into three segments: (1) the segment distal to the right cuspid, (2) the segment distal to the left cuspid, and (3) the segment anterior to the bicuspids. The buccal and lingual sides of each segment were scored. One debris and one calculus score was given to each segment by selecting the score of the greatest surface area covered by either debris or calculus, respectively. The scoring criteria are the same as the OHI criteria that were later published by Greene \& Vermillion (1960).

Measurement of degree of gingival inflammation was carried out by using both the PI and PDI criteria. Gingivitis data could not be analyzed separately in this report, because, in both indices, a gingivitis score was not recorded when pockets (more than $3.0 \mathrm{~mm}$ ) were diagnosed.

The data on loss of periodontal attachment from the 1958 New Mexico adult survey have not been previously analyzed. Reports describing some of the results of the survey have been published (Smith \& Striffler 1962, Guyer \& Striffler 1964, Sheiham \& Striffler 1970a, 1970b, Gilmore 1970).

\section{The 1984 Deming-Lordsburg survey of adults}

In 1984, two communities in New Mexico were selected as sites for a study of the effects of severe dental fluorosis on oral health status. Lordsburg (3.5 ppm F) and Deming (0.7 ppm F) were selected because of the availability of many individuals who were long-term residents of the two communities.

Recruitment of eligible residents was carried out by local coordinators. The 
selection process required that the individuals be between the ages of $30-65 \mathrm{yr}$, were born in their respective communities, had been continuous residents for at least the first $6 \mathrm{yr}$ of their life in the community, and had consumed community water during that time. These data were self-reported. More than $80 \%$ of the eligible residents contacted consented to participate in the study. Out of the 377 dentate residents who were examined, 372 had loss of attachment measurements and therefore were included in this analysis (Table 1).

The difference in the prevalence of the following oral conditions was evaluated during the project: fluorosis, coronal and root caries, attrition, abrasion, gingival recession and bleeding, pocket depth and loss of periodontal attachment, presence of plaque and calculus, and range of mandibular movements (maximum opening, protrusion and lateral movements, overjet, and overbite). Three bitewing radiographs, one anterior and two posterior films, were exposed. The examinees also completed a questionnaire investigating the presence of symptoms of temporomandibular joint disorders, demographic characteristics, ethnic origin, and use of dental services.

Four dental examiners participated in the project with each examiner measuring different oral conditions. For the oral conditions included in this report, two examiners collected all the data. The first examiner measured the degree of plaque accumulation on the six index teeth of Ramfjord (1959) using a dichotomous scoring method: when plaque was visible or was removed when the cervical margin was contacted by an explorer, a positive score was given, and when no plaque was detected on the index tooth, a zero score was awarded.

Following measurement of plaque accumulations, the first examiner proceeded with measurement of the tendency for gingival bleeding on the facial and mesiofacial tooth surfaces. Assessment of the presence of gingival bleeding was carried out by running a $\mathrm{Hu}$ Friedy no. 11 periodontal probe in the gingival crevice of the index teeth. The examiner first checked the upper index teeth and returned to check whether there was bleeding at the midpoint of the facial surface and mesiofacial line angle of the gingival margin. The procedure was then repeated on the lower arch. When bleeding was noticed, the examiner awarded the tooth site a posi-

Table 4. Mean number of tooth sites per person with specified amount of loss of periodontal attachment, by age, 1984 Deming-Lordsburg survey of adults

\begin{tabular}{cccccc}
\hline Age & $\mathrm{N}$ & $1-3 \mathrm{~mm}$ & $4-6 \mathrm{~mm}$ & $7+\mathrm{mm}$ & $\begin{array}{c}\text { Mean no. of tooth } \\
\text { sites examined } \dagger\end{array}$ \\
\hline $27-46$ & 252 & 7.3 & 0.8 & 0.18 & 11.77 \\
$47-74$ & 120 & 6.9 & $2.0^{*}$ & $0.75^{*}$ & 11.21 \\
\hline
\end{tabular}

* Mean loss of those aged 47-74 is significantly higher than those aged $27-46 \mathrm{yr}, \mathrm{p}<0.0001$. $\dagger$ Includes tooth sites with no loss of periodontal attachment.

tive score, while when no bleeding was noticed, a score of zero was awarded.

The second examiner assessed the presence or absence of supragingival and subgingival calculus and measured the loss of periodontal attachment. In assessing the presence of calculus, the examiner first probed using a $\mathrm{Hu}$-Friedy no. 17 explorer for subgingival calculus on each index tooth. When subgingival calculus was not detected, the examiner proceeded with the visual and tactile inspection for supragingival calculus.

Loss of periodontal attachment was measured to the nearest millimeter using a $\mathrm{Hu}$-Friedy no. 11 periodontal probe on the midfacial and mesiofacial line angles following the technique described by Ramfjord (1959).

\section{Statistical analysis}

Testing for the difference in the mean number of teeth with loss of periodontal attachment between age, gender, education, and ethnic group was carried out using the analysis of variance and Bonferroni t-tests. Differences between the groups in the percentage of individuals with at least one tooth site with advanced loss of periodontal attachdence intervals. The logistic regression ment was carried out using 95\% confi-

model was used to correlate the probability of an individual having at least one tooth site with advanced loss of periodontal attachment with age, gender, education, ethinic group, presence of plaque, calculus, and gingival bleeding. All analyses were conducted using the Michigan Interactive Data Analysis System (MIDAS).

\section{Findings \\ Loss of periodontal attachment by age, gender, race, and education: The $1958 \mathrm{New}$ Mexico survey of adults}

The mean number of teeth with $7.0+$ $\mathrm{mm}$ loss of attachment increased significantly with age $(p<0.05)$ (Table 2). Only 0.3 and $1.5 \%$ of the teeth with loss of attachment had more than $7.0 \mathrm{~mm}$ of loss in those aged 17-26 and 27-46 $\mathrm{yr}$, respectively. The percentage of individuals with at least one tooth site with $7.0+\mathrm{mm}$ loss of attachment increased significantly from only $2.4 \%$ in those aged $17-26$ to $14.1 \%$ in those adults aged 27-46 $(\mathrm{p}<0.05)$ (Table 3$)$.

A significantly higher percentage of males had teeth with advanced loss of attachment (Table 3). Hispanics had a significant higher prevalence of advanced loss than Anglos $(\mathrm{p}<0.05)$, and so did those with 8 yr or less of edu-

Table 5. Percentage of persons with at least one site with a specified amount of loss of periodontal attachment, by selected characteristics, 1984 Deming-Lordsburg survey of adults

\begin{tabular}{lrrrr}
\hline Characteristic & $\mathrm{N}$ & $1-3 \mathrm{~mm}$ & $4-6 \mathrm{~mm}$ & $7+\mathrm{mm}$ \\
\hline Age & & & & \\
$27-46$ & 252 & 100.0 & 32.1 & 8.3 \\
$47-74$ & 120 & 95.0 & 71.7 & 34.2 \\
Gender & & & & \\
Male & 129 & 96.1 & 51.2 & 21.7 \\
Female & 243 & 99.6 & 41.6 & 14.0 \\
Ethnic Group & & & & \\
Hispanic & 297 & 98.3 & 46.8 & 17.8 \\
Other & 72 & 98.6 & 37.5 & 11.1 \\
Education (yr) & & & & \\
1-8 & 37 & 94.6 & 73.0 & 37.8 \\
9-11 & 73 & 97.3 & 50.7 & 20.5 \\
12 & 165 & 99.4 & 41.8 & 12.7 \\
College & 94 & 100.0 & 34.0 & 11.7 \\
\hline
\end{tabular}


cation when compared with those with more advanced education $(\mathrm{p}<0.05)$.

\section{The 1984 Deming-Lordsburg survey of adults}

Individuals aged 47-74 yr in Deming and Lordsburg, New Mexico, had significantly higher mean numbers of tooth sites with $7.0+\mathrm{mm}$ loss of attachment than adults aged 27-46 (Table 4). Only 1.5 and $6.7 \%$ of the tooth sites examined had $7.0+\mathrm{mm}$ loss in those 27-46 and 47-74 yr of age, respectively, while a significantly higher percentage of tooth sites had $1.0-3.0$ or $4.0-6.0 \mathrm{~mm}$ loss of periodontal attachment.

Table 5 presents the percentages of individuals with at least one tooth site affected by loss of periodontal attachment by degree of loss, and selected demographic variables. Only $8.3 \%$ of those 27-46 yr of age had one tooth site or more with $7.0+\mathrm{mm}$ loss. The percentage increased significantly to 34.2 in those $47-74$ yr old.

In 1984, male residents of Deming and Lordsburg, New Mexico, did not have a significantly higher prevalence of advanced loss of attachment than females. Those with only grade school education had a significantly higher prevalence of 4.0-6.0 and 7.0+ $\mathrm{mm}$ loss than those who had completed high school or attended college. No significant differences were found between $\mathrm{Hi}$ spanics and other residents of the two cities.

\section{Loss of periodontal attachment and status of oral hygiene and gingivitis The 1958 New Mexico survey of adults: Plaque accumulations}

The mean number of teeth with advanced loss of attachment increased significantly with increases in Debris Index (DI) scores $(\mathrm{p}<0.0001)$ (Table 6). Those individuals having teeth with high DI scores (larger than 1) had an average of 0.4 teeth with $7.0+\mathrm{mm}$ loss, whereas those with no or little plaque accumulations had an average of only 0.1 tooth sites with advanced loss.

\section{Calculus scores}

Significantly higher mean numbers of teeth with loss of attachment of different severities were found in those with abundant calculus accumulations (Table 6). The differences between those with low calculus accumulations (Cal- culus Index [CI] score of 0-0.5) and lations (CI score larger than 1.0) were similar to differences found between those with low and high plaque accumulations.

\section{The 1984 Deming-Lordsburg survey of adults: Plaque accumulations}

Those residents of Deming and Lordsburg, New Mexico, with some plaque accumulation had a significantly higher mean number of tooth sites with advanced loss of periodontal attachment (Table 7) $(\mathrm{p}<0.0001)$.

\section{Subgingival calculus}

The mean number of tooth sites with advanced loss of attachment was lower in those with 0-3 teeth with subgingival calculus when compared with those with 46 teeth with subgingival calculus, but the difference was not statistically significant - perhaps because of the small number of individuals who were free from subgingival calculus (Table 7).

\section{Gingival bleeding}

Those with gingival bleeding had a higher mean number of tooth sites with advanced loss of attachment than those with no tooth site with gingival bleeding, but the difference was not statistically significant.

\section{Multivariate analysis}

To test for the joint associations between two or more risk factors and loss of attachment, logistic regression analysis was used. The logistic regression analysis, in this study, correlates the probability of having at least one tooth or tooth site with $7.0+\mathrm{mm}$ loss of attachment and the joint occurrence of those with abundant calculus accumu-

the different risk factors measured in both studies.

In the New Mexico adults examined in 1958, the two risk factors that remained significant even after accounting for the confounding effect of other risk factors were age and degree of calculus accumulation (Table 8). Plaque accumulation did not remain a significant risk factor for the probability of having $7.0+\mathrm{mm}$ loss of attachment. There was, however, a high correlation between the presence of plaque and calculus in the persons examined in 1958 (Pearson correlation coefficient was 0.6). All risk factors included in this analysis explained only about $7 \%$ of the variability in the probability of having at least one tooth with $7.0+\mathrm{mm}$ loss of attachment.

In the residents of Deming and Lordsburg, the probability of having at least one tooth site with $7.0+\mathrm{mm}$ loss of attachment was associated with age and number of teeth with plaque accumulation (Table 8). Subgingival calculus did not remain a significant risk factor when other factors were accounted for. All the risk factors included in the analysis explained about $9 \%$ of the variability in the probability of having advanced loss of attachment.

\section{Discussion}

The intent of this analysis was not to compare the prevalence of disease in these two samples directly, nor to look for secular trends in the prevalence of advanced loss of attachment, because of the many differences between the two studies. When presented with two broadly similar samples, however, it is difficult to resist the temptation to compare disease rates. Data on loss of periodontal attachment in Americans are scarce. As has previously been stated, most surveys in the United States have

Table 6. Mean number of teeth per person with advanced loss of periodontal attachment, by DI and CI Scores, 1958 New Mexico survey of adults

\begin{tabular}{lrccc}
\hline & $\mathrm{N}$ & Mean & SD & $\begin{array}{c}\text { Mean no. of tooth } \\
\text { sites examined }\end{array}$ \\
\hline $\begin{array}{l}\text { DI Scores } \\
0-0.5\end{array}$ & 296 & 0.1 & 0.8 & 25.3 \\
$0.6-1.0$ & 1014 & 0.2 & 0.9 & 25.3 \\
$1.1-3.0$ & 664 & 0.4 & 1.5 & 25.6 \\
CI Scores & & & & \\
$0-0.5$ & 400 & 0.03 & 0.2 & 25.7 \\
$0.6-1.0$ & 335 & 0.2 & 0.9 & 25.7 \\
$1.1-3.0$ & 1239 & 0.4 & 1.3 & 25.2 \\
\hline
\end{tabular}


Table 7. Mean number of tooth sites per person with advanced loss of periodontal attachment by presence of plaque, subgingival calculus, and gingival bleeding, 1984 Deming-Lordsburg survey of adults

\begin{tabular}{lcccc}
\hline & N & Mean & SD & $\begin{array}{c}\text { Mean no. of tooth } \\
\text { sites examined }\end{array}$ \\
Plaque & & & & \\
No & 121 & 0.1 & 0.06 & 11.9 \\
Yes & 251 & 0.5 & 1.7 & 11.4 \\
Subgingival & & & & \\
Calculus & & & & 11.5 \\
0-3 Teeth & 27 & 0.1 & 0.07 & 11.9 \\
4-6 Teeth & 345 & 0.4 & 1.3 & 10.4 \\
Gingival Bleeding & & & & 11.7 \\
No & 106 & 0.2 & 1.2 & \\
Yes & 266 & 0.4 & 0.3 & \\
\hline
\end{tabular}

used the PI criteria to measure periodontal disease, resulting in little information about the prevalence of pockets of different depths or loss of periodontal attachment. The 1985-86 National Institute of Dental Research Survey of Adults may provide, for the first time, national data on loss of periodontal attachment. Those data may be used to investigate further the distribution of advanced loss of periodontal attachment identified in this study.

Another reason why the data from two surveys - which were carried out in the same state but $26 \mathrm{yr}$ apart - are presented here is the availability of loss of periodontal attachment information on all teeth present in the mouth in the 1958 data set and on six index teeth in the 1984 study. Six index teeth were measured in 1984 because of time limitations and to reduce discomfort to the participants in the study who received an extensive evaluation of their oral health status by four examiners. The use of these six index teeth rather than all teeth present in the mouth may underestimate the prevalence of advanced loss of attachment (Ismail et al. 1985). Regardless of the underestimation that could result from using these six index teeth, the analysis of loss of attachment data from both studies showed that the percentage of tooth sites and persons, 27-46 yr of age, with advanced loss of periodontal attachment were quite similar in 1958 and 1984. In those older than $46 \mathrm{yr}$, only $6.7 \%$ of the teeth had advanced loss of periodontal attachment. Tooth sites with advanced loss clustered in $34.2 \%$ of those examined. Further analysis of the intraoral distribution of loss of attachment showed that in $52 \%$ of the adults one tooth site was affeced, while in $25 \%$ two sites were affected by advanced loss.

These findings question many of the long-held views concerning the inevitability of periodontal destruction and loss of teeth. Periodontal disease has been suggested as the main cause of loss of teeth in adults (American Academy
Table 8. Logistic regression coefficients and their standard errors, 1958 and 1984 surveys

\begin{tabular}{lcc}
\hline Variable Name & B & SE \\
\hline 1958 New Mexico survey of adults & & \\
Age & 0.13 & $0.01^{*}$ \\
Gender & -0.29 & 0.17 \\
Education & 0.001 & 0.09 \\
Ethnic Group & -0.05 & 0.06 \\
Plaque & 0.41 & 0.26 \\
Calculus & 0.87 & $0.15^{*}$ \\
1984 Deming-Lordsburg survey of adults & & \\
Age & 1.63 & $0.33^{*}$ \\
Gender & -0.33 & 0.33 \\
Ethnic Group & -0.13 & 0.47 \\
Education & -0.15 & 0.18 \\
Plaque & 0.27 & $0.09^{*}$ \\
Calculus & -0.08 & 0.11 \\
Gingival Bleeding & 0.09 & 0.06 \\
\hline
\end{tabular}

*Logistic regression coefficients are significantly different from zero. of Periodontology, 1982). Such a conclusion has perhaps been reached when cross-sectional data of tooth loss and of periodontal disease are used to predict longitudinal patterns of both conditions. As a result, the concurrent increase of the prevalence of both conditions with age was considered a good indicator of the presence of a cause-andeffect association. In a survey involving 370 Finnish dentists who recorded the reason for every extraction they performed in 16-yr-old or older patients during the course of 1 month, Ainamo et al. found that periodontal disease was the reported reason for only $18.3 \%$ of the extractions (Ainamo et al. 1983). The results of the Ainamo et al. study support those reported more than $40 \mathrm{yr}$ ago by Allen, who found that only $16 \%$ of 1167 consecutive registrants at a dental school clinic required extractions because of periodontal disease (Allen 1944). Though both surveys may have a number of flaws, their data allow the popular conclusion - that periodontal disease is the major reason for tooth loss - to be questioned.

Other risk factors were also correlated with advanced loss of attachment in this study. Differences did emerge in the findings of the analysis of data collected during both surveys. Males, Hispanics, and people with only primary school education had a significantly higher mean number of teeth with advanced loss of attachment in 1958, whereas only educational status was a significant demographic risk factor for advanced loss of attachment in the 1984 data. The 1984 sample was collected from a more homogenous group of individuals who were lifetime residents of two nearby communities in New Mexico. Because of the small degree of variability among those examined in 1984, the associations found were weaker than those found in the 1958 data which included a more heterogeneous mix of individuals from all over the state of New Mexico. In 1984 , there were no statistically significant differences in the mean loss of periodontal attachment nor in the percentage of adults with advanced loss between the residents of the optimally fluoridated and high fluoride (3.5 ppm) communities.

Presence of plaque was a significant predictor of advanced loss of attachment in both the 1958 and 1984 data. Because of the almost linear association between plaque and calculus in the 1958 data, calculus was also associated with 
advanced loss of attachment. The independent association of each risk factor with advanced loss of attachment, however, cannot be separated in these data. In the 1984 study, calculus was measured using a new scoring method that gave a higher weight to the presence of subgingival calculus, and did not record supragingival calculus when subgingival calculus was present. This new scoring method was insensitive to different degrees of calculus accumulation in the 1984 sample because almost $97 \%$ of the individuals had more than one index tooth with subgingival calculus. The weak correlation between subgingival calculus and advanced loss of attachment in the 1984 data may have resulted again from this universal presence of subgingival calculus.

Another risk factor included in this analysis was gingival bleeding. In the 1958 study, gingivitis was not scored when loss of attachment or pockets were present, a drawback of both the PI and PDI indices. In the 1984 study, presence or absence of gingival bleeding around the index teeth was recorded. Those with at least one tooth site with gingival bleeding had about twice as many tooth sites with advanced loss of attachment than those with no gingival bleeding. Gingival bleeding was prevalent in those with and without advanced loss of attachment, suggesting that the specificity of this risk factor in differentiating between those with and without advanced periodontal destruction may be low. The results of the logistic regression analysis support this conclusion: only $7-10 \%$ of the variability of the presence of advanced loss of attachment was explained by all the risk factors included in this analysis.

In conclusion, the analysis presented here demonstrates that when using loss of attachment measurements, the prevalence of severe periodontal disease was quite low, an observation in agreement with recent studies of the prevalence and distribution of advanced periodontal disease. The inability of the PI and other periodontal indices to describe adequately the intraoral distribution of disease suggests that, in the future, more sensitive and specific methods of measurement, such as amount of loss of periodontal attachment and gingival bleeding, should be used. In addition, new questions about periodontal diseases and their natural history will require new research strategies. One strategy may be case-control studies to help identify risk factors beyond oral hygiene, age, and gender. Another may be longitudinal studies to determine the progression of disease and to search for secular trends in disease occurrence.

\section{Acknowledgments}

The 1958-59 research was supported in part by grant D-681 from the Public Health Service, National Institutes of Health. The 1984 research was supported in part by grant NO-1-DE-32443, Public Health Service, National Institutes of Health, National Institute of Dental Research.

\section{References}

Ainamo, J., Sarkki, L., Kuhalampi, M. L., Palolampi, L. \& Piirto, O. 1983. The frequency of periodontal extractions in Finland. Community Dental Health 1 (3): 165-172.

Allen, E. F. 1944. Statistical study of primary causes of extraction. Journal of Dental Research 23: 453-458.

American Academy of Periodontology. 1982. Proceeding from State of the Art Workshop. Journal of Periodontology 53 (8): 477-501.

Baelum, V., Fejerskov, O. \& Karring, T. 1986. Oral hygiene, gingivitis, and periodontal breakdown in adult Tanzanians. Journal of Periodontal Research 21: 221-232.

Beck, J. D., Lainson, P. A., Field, H. M. \& Hawkins, B. F. 1984. Risk factors for various levels of periodontal disease and treatment needs in Iowa. Community Dentistry and Oral Epidemiology 12: 17-22.

Burt, B. A., Ismail, A. I. \& Eklund, S. A. 1985. Periodontal disease, tooth loss, and oral hygiene among older Americans. Community Dentistry and Oral Epidemiology 13: 93-96.

Dean, H. T. 1942. The investigation of physiological effects by the epidemiological method. p. 23-31. (In Moulton, F. R., ed. 1942. Fluorine and dental health. AAAS Publication).

Gilmore, N. D. 1970. An epidemiological investigation of vertical osseous defects in periodontal disease. Dissertation. The University of Michigan, Ann Arbor, Mi.

Greene, J. C. \& Vermillion, J. R. 1960. Oral hygiene index: a method for classifying oral hygiene status. Journal of the American Dental Association 61: 172-179.

Guyer, M. E. \& Striffler, D. F. 1964. The reported frequency of toothbrushing as related to the prevalence of periodontal diseases in New Mexico. Journal of Public Health Dentistry 24: 62-75.

Hughes, J. T., Rozier, R. G. \& Ramsey, D. L. 1982. Natural history of dental diseases in North Carolina, 1976-1977. Durham, N. C: Carolina Academic Press.

Hugoson, A., Jordan, T. 1982. Frequency distribution of individuals aged $20-70$ years according to severity of periodontal disease. Community Dentistry and Oral Epidemiology 10: 187-192.

Ismail, A. I., Eklund, S. A., Burt, B. A. \& Calderone, J. J. 1986. Prevalence of deep periodontal pockets in New Mexico adults aged 27 to 74 years. Journal of Public Health Dentistry 46: 199-206.

National Center for Health Statistics, 1979. Basic data on dental examination findings of persons 1-74 years. United States, 1971-74. Vital and Health Statistics: Series 11, no. 214, DHEW publication 79-1662.

National Center for Health Statistics, 1965. Periodontal disease in adults, United States, 1960-62. Vital and Health Statistics, PHS publication no 1000 , Series 11 , no. 12.

Ramfjord, S. P. 1959. Indices for prevalence and incidence of periodontal disease. Journal of Periodontology 30: 51-59.

Reddy, J., Parker, J. R., Africa, C. W. \& Stephen, L. X. G. 1985. Prevalence and severity of periodontitis in a high fluoride area in South Africa. Community Dentistry and Oral Epidemiology 13: 108-112.

Russell, A. L. 1965. A system of classification and scoring for prevalence surveys of periodontal disease. Journal of Dental Research 35: 350-359.

Sheiham, A. \& Striffler, D. F. 1970a. A comparison of four epidemiologic methods of assessing periodontal disease. I. Population findings. Journal of Periodontal Research 5: 148-154.

Sheiham, A. \& Striffler, D. F. 1970b. A comparison of four epidemiologic methods of assessing periodontal disease. II. Test of periodontal indices. Journal of Periodontal Research 5: 155-161.

Smith, A. J. \& Striffler, D. F. 1962. The reported frequency of toothbrushing as related to the prevalence of dental caries in New Mexico. Journal of Public Health Dentistry 23: 159-175.

\section{Address:}

Amid I Ismail, BDS, MPH, Dr. PH

Faculty of Dentistry

3640 University Street

McGill University

Montreal

Quebec, H3A 2B2

Canada 
This document is a scanned copy of a printed document. No warranty is given about the accuracy of the copy. Users should refer to the original published version of the material. 